\title{
ON THE STRUCTURE OF CONTINUA*
}

\author{
BY G. T. WHYBURN
}

1. Introduction. The notion of a continuum, according to present usage of the term, is both an abstraction and a generalization of the more classical concept of the continuum of real numbers. The essential properties taken from the line or interval are its closedness and its connectedness. A set of points is said to be closed if it contains all of its limiting points; and, following the Lennes-Hausdorff definition, a set is connected provided that however it be divided into two disjoint subsets, one of these must contain a limiting point of the other. Loosely speaking, a set is connected provided it all hangs together in one piece. Thus an interval, a line, or a circle is connected, whereas a set consisting of two distinct intervals, or of a circle and an interval not intersecting it, or of an interval and a point not on it would not be connected. Combining these two properties, then, we understand by a continuum any set which is both closed and connected. Thus all properties of the linear continuum which have to do with its linearity or with the order and arrangement of its points have been rejected or, rather, reserved for more specialized topological concepts. Hence not only are sets such as an interval, circle, lemniscate, and sphere included among the continua but also many less orthodox sets such as the curve $y=\sin (1 / x)$ together with the interval $(-1,1)$ of the $y$ axis, or the set consisting of a circle and a spiral approaching it asymptotically, and a host of even more complicated figures.

I shall not attempt to bring in review all of the many valuable results proved in recent years which may be relevant to the title I have selected for my address. Instead I shall limit my remarks to certain lines of investigation which have been most closely related to my own work and which lead to some of the most interesting and important unsolved problems in the field of topology.

* An address delivered at the meeting of this Society on September 13, 1935, in Ann Arbor, by invitation of the Committee on Program. 
Inasmuch as the principal interest of the results to be discussed lies in their concrete visual character rather than in the great generality or abstractness of the spaces in which they hold, for simplicity I shall suppose once for all that the sets I consider all lie in a compact metric space, or even in a bounded portion of a euclidean space, although in nearly all cases this is an unnecessary restriction. A continuum, then, will be a closed, connected, and compact set of points.

2. Cut Points. My paper is devoted largely to theorems grouped around the notion of a cut point of a continuum and the key which this notion gives to the structure of a continuum through its decomposition into cyclic elements. The point $p$ of a continuum $M$ is called a cut point of $M$ provided the set $M-p$ is not connected. Thus $M-p$ falls into at least two disjoint sets neither of which contains a limiting point of the other. For example, any interior point of an arc is a cut point, the double point of a lemniscate is a cut point but is its only cut point, all points on the continuum constructed above using the curve $y=\sin (1 / x)$ except the end point and points on the limiting set are cut points, and no point of a simple closed curve is a cut point.

This type of point was first studied by R. L. Moore [1]* and S. Mazurkiewicz [1], * each of whom proved theorems from which it follows that no simple closed curve can contain more than a countable number of cut points of any locally connected continuum $†$ containing it. Moore's result is considerably more general and states that if $K$ is any subcontinuum of an arbitrary continuum $M$, then all save at most a countable number of the cut points of $M$ which belong to $K$ are cut points also of $K$. This theorem in turn has been shown to hold for arbitrary connected sets $M$ and $K$ by Zarankiewicz [1]. Moore also obtained a characterization in terms of cut points of the class of continua called dendrites, that is, the locally connected continua containing no simple closed curves. As a result of a theorem proved later by R. L. Wilder [1], this may be stated as

* Numbers in brackets refer to the bibliography at the end of the paper.

$\dagger$ A continuum $M$ is locally connected provided each point of $M$ is contained in arbitrarily small neighborhoods whose common part with $M$ is connected. 
follows: In order for a continuum $D$ to be a dendrite it is necessary and sufficient that $D$ consist wholly of cut points and end points. *

Now an excellent account of the results concerning cut points which were known up to that time was given in 1927 by J. R. Kline in an address before this Society which has subsequently been published in the Bulletin (see Kline [1]). Hence I shall content myself with mentioning only two more recent results which seem to be of fundamental and central significance. The first of these might well be called the Cut Point-Order Theorem. It states that all save a countable number of the cut points of any continuum whatever must be points of MengerUrysohn ordert 2 of that continuum (see G. T. Whyburn [1]). Thus in particular it follows that the branch points of a dendrite must be countable; and, indeed, the points of any continuum which cut it into more than two pieces must be countable. Also, since any continuum is locally connected at every point of finite order, it follows that any continuum is locally connected at all except possibly a countable number of its cut points.

The second result has to do with the Borel classification of the set $G$ of all cut points of a continuum $M$. Zarankiewicz [1] has shown that if $M$ is locally connected, the set $G$ of all of its cut points is an $F_{\sigma}$-set, that is, a set which is the sum of a countable number of closed sets. Later it was shown in one of my own papers (see Whyburn [2]) that the set of all cut points of an arbitrary continuum is a Borel set of the class $G_{\delta \sigma}$, that is, a set which is the sum of a countable number of $G_{\delta}$-sets, where a $G_{\delta}$-set in turn is one which is the product of a countable number of open sets. From these results it follows that if the set $G$ of all cut points of a continuum $M$ is uncountable, it necessarily contains a perfect set; and thus in any case the set $G$ is either vacuous, finite, countable, or of the power of the continuum.

* By an end point of a continuum $M$ we will understand a point $p \epsilon M$ which is contained in arbitrarily small neighborhoods whose boundaries intersect $M$ in just one point, that is, a point of $M$ of Menger-Urysohn order 1 . That this definition is equivalent to earlier definitions used by various authors was proved by H. M. Gehman [1].

$\dagger$ The point $p$ of a continuum $M$ is a point of Menger-Urysohn order $n$ of $M$ provided $n$ is the least integer such that $p$ is contained in arbitrarily small neighborhoods whose boundaries intersect $M$ in at most $n$ points. See Menger [1] and Urysohn [1]. 
3. Cyclic Elements. The principal feature of cut points with which I shall deal is the insight they give into the structure of a locally connected continuum through the decomposition of such a continuum into cyclic elements.

Let $S$ designate any definite locally connected continuum. Then the cyclic elements of $S$ are (a) the cut points of $S$, (b) the end points of $S$ (that is, the points of order 1) and (c) the subsets $C$ of $S$ which are connected and have no cut point and are maximal in $S$ relative to this property (see Whyburn [3] and [4]). The cyclic elements of type (c) are called true cyclic elements or non-degenerate cyclic elements, since the elements of the other two types reduce to single points. There are other equivalent ways of defining the cyclic elements of $S$ (see Moore [2], and Kuratowski [1] and Whyburn), but I have adopted this definition here since in this form it admits of extension to what we may call $n$-dimensional or $n$ th-order cyclic elements of an arbitrary continuum.

For example, in the continuum consisting of two disjoint circles joined by an arc, the two circles are true cyclic elements and every other remaining point is itself a degenerate cyclic element. A sphere has only one cyclic element, namely, the sphere itself. A lemniscate has two true cyclic elements and one cut point. In the continuum consisting of an infinite sequence of tangent spheres converging to a single point, the spheres are true cyclic elements, the points of tangency are cut points (and thus are cyclic elements) and the point to which the spheres converge is an end point (also a cyclic element). The cyclic elements of a dendrite are all individual points, so that it has no true cyclic elements.

Now let us consider briefly some of the general properties of the cyclic elements of a locally connected continuum $S$. In the first place, $S$ is the sum of its cyclic elements, that is, every point of $S$ is either a cut point, an end point, or a point of at least one true cyclic element. Furthermore, the true cyclic elements of $S$ are countable, in fact there are at most a finite number of them of diameter greater than any preassigned positive quantity, and no two of them intersect in more than one point. If two of them do have a common point, this point must be a cut point of $S$, so that the intersection of two cyclic elements is either vacuous or is itself a cyclic element. Also each 
true cyclic element $C$ is itself a locally connected continuum and moreover $C$ is cyclicly connected (see Whyburn [4] and Ayres [1]) in the sense that any two points of $C$ lie together on a simple closed curve in $C$. If $Z$ is an arbitrary connected subset of $S$, the intersection $Z \cdot C$ of $Z$ with an arbitrary cyclic element is either vacuous or connected. Thus it follows in particular that any arc in $S$ whose end points belong to $C$ must itself lie wholly in $C$. From this property it results that there can be no closed ring of cyclic elements.

Hence, with respect to its cyclic elements, the continuum $S$ has a structure analogous to that of a dendrite. There are many other properties of cyclic elements and of $S$ relative to its cyclic elements which strengthen this analogy. For example, a dendrite is characterized among the locally connected continua by the property of containing one and only one simple arc joining any two of its points. Correspondingly, for any locally connected continuum $S$, there is one and only one simple chain (see Whyburn [3]) of cyclic elements joining any two given elements. Also, any connected subset of a dendrite is arcwise connected; and correspondingly, any connected set of cyclic elements of $S$ is arcwise connected and also cyclic chainwise connected.

It is notable that sets of this type, that is, connected sets of cyclic elements, as well as closed and connected sets of cyclic elements, present many interesting phenomena. For example, Ayres has shown (see Ayres [2]) that they may be identified with the sets which he has called arc-curves. If $K$ is any subset of $S$, then by the arc-curve $M(K)$ is meant the set of all points $x$ of $S$ which lie on $\operatorname{arcs} a x b$ in $S$ joining some two points $a, b \in K$. Ayres shows that any arc-curve is a connected set of cyclic elements and conversely any connected set $H$ of cyclic elements is an arc-curve, in fact we have $M(H) \equiv H$.

4. Cyclicly Extensible and Reducible Properties. It may well be said that it is to this same "dendritic" structure of an arbitrary locally connected continuum relative to its cyclic elements that we owe the principal applications of the cyclic element notion. The fruitfulness of this notion in the study of the structure of locally connected continua is due in large measure to the fact that so many questions concerning locally 
connected continua of various types can be reduced to the same questions concerning the individual cyclic elements of those continua. In other words, there are a large number of properties $P$ which are cyclicly extensible in the sense that if each cyclic element has property $P$, then the whole continuum $S$ has property $P$. Similarly a property $P$ is cyclicly reducible provided that if $S$ has property $P$, so also does each cyclic element of $S$. Thus $P$ is cyclicly extensible if

$$
(P \text { in each } C) \rightarrow(P \text { in } S) \text {; }
$$

$P$ is cyclicly reducible if

$$
(P \text { in } S) \rightarrow(P \text { in each } C) \text {. }
$$

Let us consider some properties of this sort. First, for a continuum $M$, let $P$ be the property of having all of its connected subsets arcwise connected. Clearly this is a property of simple sets such as arcs, simple closed curves, $\theta$-curves, that is, curves like the letter $\theta$, and in fact of linear graphs in general. Furthermore, this property is cyclicly extensible and reducible. Consequently any locally connected continuum $S$ every true cyclic element of which is, say, a simple closed curve, has this property. Now suppose we had this question: Given a locally connected continuum $M$ in the plane, let $S$ be the boundary of any complementary domain of $M$; then is it true that every connected subset of $S$ is arcwise connected? This question was answered in the affirmative in 1923 by R. L. Wilder (see Wilder [1]) by other methods. However, we can answer it readily in the following way. First it is known that $S$ is locally connected and that every true cyclic element of $S$ is a simple closed curve. Then since each cyclic element of $S$ has the property in question and this property is cyclicly extensible, it results that $S$ itself has this property. The same reasoning would apply, of course, to the case where every true cyclic element is a $\theta$-curve or in fact is a linear graph of any sort. This illustrates the usefulness of the cyclic element decomposition. For we are here able to reduce the problem to the same problem for the cyclic elements by virtue of the cyclic extensibility of the property involved, and then to solve it for the cyclic elements since their structure is markedly simpler than is that of the whole continuum. 
Cyclicly extensible properties, then, seem to be of fundamental importance. In a report on cyclic elements published in 1930 by C. Kuratowski and myself there were listed some fourteen non-trivial properties of this sort, and since that time at least as many more significant ones have been discovered. I shall discuss briefly here only three or four which seem particularly interesting.

(a) Separation of the plane and of $n$-space. In the first place let us consider the property $P$ of failing to separate the plane, and let us suppose our continuum $S$ lies in a plane. This property is cyclicly extensible and reducible as was shown in a paper of mine published in 1928 (see Whyburn [3]). Thus if no cyclic element of $S$ separates the plane, neither does $S$; and conversely if $S$ does not separate the plane, neither does any cyclic element of $S$. Now any locally connected plane continuum $C$ which has no cut point and fails to separate its plane must be a closed 2-cell, that is, it is homeomorphic with a circle plus its interior. Thus we have the result, that in order that a locally connected plane continuum $S$ should fail to separate its plane it is necessary and sufficient that every true cyclic element of $S$ be a closed 2-cell (see Whyburn [3]). This type of continuum $S$ has been called a base set by C. B. Morrey [1] who has used it extensively in his recent work on the theory of surfaces.

Now it is well known that the property of not separating the plane may be stated as an intrinsic property of $S$ by saying that the one-dimensional connectivity or Betti number of $S$ is 0 . This raises the question as to whether the property of having a vanishing first Betti number is cyclicly extensible in an arbitrary locally connected continuum $S$, whether $S$ lies in a plane or not. This is indeed true, as was shown by Borsuk (see Borsuk [1]). I shall consider this result in more detail later since it also appears as a special case of a much more general formula for Betti numbers of all dimensions. Borsuk also showed (Borsuk [2]) that the property of failing to separate $n$-space is cyclicly extensible when $S$ is imbedded in the euclidean $n$-dimensional space, for any $n$.

(b) Fixed point property. Consider next the property of a continuum $A$ to have a fixed point under every continuous transformation of $A$ into a subset of itself. A point $x$ is said to be a fixed point of a transformation $T(A)=B$ provided $T(x)=x$; and 
if for every single-valued [though not necessarily (1-1)] and continuous transformation $T(A)=B$, where $B \subset A$, there is at least one fixed point, then $A$ has our property. Now a set such as an interval, a 2-cell, or a $T$-shaped curve will have this property; and it was shown by Brouwer that an $n$-cell has it. On the other hand a circle would not have it, since a simple rotation of a circle through an angle of $\pi$ leaves no point fixed.

Now it was shown by Sherrer [1] that every dendrite has the fixed point property. This result in a way suggests that the fixed point property might be cyclicly extensible; and it was shown by W. L. Ayres [3] that if we restrict ourselves to topological transformations in defining the fixed point property (that is, to transformations which are both (1-1) and continuous), then this property is extensible. In fact Ayres proves in general that if $T(S)=R$ is any topological transformation of a locally connected continuum $S$ into a subset $R$ of itself, then there is at least one cyclic element $C$ of $S$ which maps into a subset of itself under $S$, that is, we have $T(C) \subset C$. From this it results of course that if each such element $C$ has a fixed point under every homeomorphism of $C$ into a subset of itself, then so also does the whole continuum $S$. It was shown independently by Borsuk [2] that even without restricting it to topological transformations, the fixed point property is cyclicly extensible. Thus not only is it a property of dendrites as shown by Sherrer but also of a continuum such as two tangent circles with their interiors or a set consisting of a circle together with its interior plus a perpendicular through its center. In fact, since by the Brouwer fixed point theorem it follows that every $n$-cell has this property, it results that any locally connected continuum every true cyclic element of which is a $k$-cell, where $k$ may vary from element to element, has the fixed point property. Thus, in particular, any base-set has this property.

(c) Curve types. As a final example, let us consider some curve types occurring in the curve theory and dimension theory as developed extensively by Urysohn [1] and Menger $[1,2]$. A continuum $M$ is said to be a regular curve provided each point of $M$ is contained in arbitrarily small neighborhoods whose boundaries intersect $M$ in only a finite set of points, and $M$ is a rational curve if these neighborhoods can be chosen so that they intersect $M$ in only a countable set of points. For example, a 
circle plus two perpendicular diameters is regular and hence also rational; a curve consisting of an interval $A B$ together with a sequence of intervals $A B_{1}, A B_{2}, \cdots$ converging to $A B$ is rational but not regular. Similarly, $M$ is said to be of dimension $\leqq n$ provided each point of $M$ has arbitrarily small neighborhoods whose boundaries intersect $M$ in a set of dimension $\leqq n-1$, where the null set has dimension -1 . Now the property of being a regular curve (Whyburn [5]), of being rational (Whyburn [6]), or of being of dimension $\leqq n$ for $n>1$ (Kuratowski [2]) are all cyclicly extensible and reducible. Thus if each true cyclic element of $S$ is a regular curve, so also is $S$; hence, in particular, if the boundary of a plane domain is locally connected, it is a regular curve, since each of its true cyclic elements is a simple closed curve. Likewise the Menger-Urysohn dimensionality of $S$ may be found by taking the maximum of the dimensionalities of the true cyclic elements.

Another important curve type is the so called hereditarily locally connected continuum, that is, a continuum $M$ having the property that every subcontinuum of $M$ is locally connected. The property of being hereditarily locally connected also is cyclicly extensible and reducible (Whyburn [3]). In fact, as Zippin [1] has noted, we may say even more, namely, that if some subcontinuum $N$ of $S$ fails to be locally connected, then there exists a true cyclic element $C$ of $S$ such that the continuum $C \cdot N$ fails to be locally connected.

5. Cyclic Elements of Higher Orders. So much for the cyclic element decomposition of a locally connected continuum. The usefulness of this decomposition leads us naturally to ask (see Wilder [2]) whether it is not possible in the first place to extend it to arbitrary continua, locally connected or not, and in the second place to carry it further and obtain a finer decomposition of a continuum in some analogous fashion into elements with respect to which the structure of the continuum is simple, though not so simple as the dendrite, and which may yield an even deeper light into the structure of the continuum. This is indeed the case; for as we shall see, it is possible to obtain, for each integer $r \geqq 0$, a decomposition of this sort of any continuum into elements which we will call $E_{r}$ which enjoy properties analogous to those of the cyclic elements and reduce, 
in case $r=0$ and in case the continuum is locally connected, to the cyclic elements themselves.

The key to these finer decompositions is furnished by the notion of a complete cycle as introduced originally by Vietoris [1] and subsequently extensively developed by Vietoris, Alexandroff, Čech, Borsuk, Lefschetz, R. L. Wilder, Pontrajagn, Kuratowski, and others. For convenience and to distinguish them from the ordinary geometric combinatorial cycles $\Gamma^{r}$, we shall follow the usual practice of calling these cycles "Vietoris cycles" and we shall designate them by $\gamma^{r}$, where $r$ denotes the dimensionality of the cycle. For our purposes we will understand by a cycle of dimension 0 , either geometric $\Gamma^{0}$ or Vietoris $\gamma^{0}$, any even number of points (0-cells). A Vietoris cycle is said to be essential if it has at least one carrier $C$ in which it is not $\sim 0$. A closed set $C$ "carries" a $\gamma^{r}$ provided all vertices of all cycles in $\gamma^{r}$ belong to $C$.

We shall call a closed set of points which carries no essential $r$-dimensional Vietoris cycle a $T_{r}$-set or simply a $T_{r}$. Thus a single point is a $T_{0}$, an arc or dendrite is a $T_{1}$, a 2-cell is a $T_{2}$, and so on; whereas a point pair is not a $T_{0}$, a circle is not a $T_{1}$, but is a $T_{r}$ for $r>1$, a sphere or torus is not a $T_{2}$, and so on.

Let $M$ denote any compact continuum. A non-degenerate subset $X$ of $M$ will be called an $E_{r}$-set in $M$ or merely an $E_{r}$, provided $X$ is not separated by any $T_{r}$ in $X$ and $X$ is maximal in $M$ relative to this property. In other words, if no $T_{r}$ in $X$ cuts $X$ and if, when $Y$ is any larger set containing $X$, some $T_{r}$ cuts $Y$, then $X$ is an $E_{r}$. The sets $E_{r}$ may be called the $r$ th order true cyclic elements of $M$ for each $r \geqq 0$. It is to be noted first, that in case $r=0$, the sets $E_{r}$ are the maximal sets in $M$ which are not separated by any $T_{0}$, that is, by any single point. Thus the sets $E_{0}$ are the maximal sets in $M$ having no cut point, so that if $M$ is locally connected they are identically the ordinary true cyclic elements of $M$. For $r=1$ consider the following example. Let $W$ be the set consisting of a torus together with a disk just fitting into it. Let $C$ be a cube or sphere attached to $W$ along a simple arc, let $Q$ be a 2-cell attached to $C$ along an arc and intersecting $W$ along another arc having nothing in common with $C$, and let $M=W+C+Q+$ any finite collection of arcs joined on in an arbitrary manner. Then the sets $E_{1}$ in $M$ are $W$ and $C$, whereas the sets $W$ and $C$ lie in the same $E_{0}$ (cyclic element), 
of $M$. For any set cutting $C$ or $W$ must contain a ring-shaped figure and thus carries a 1 -cycle, whereas any larger continuum in $M$ containing $C$ or $W$ is cut by a $T_{1}$.

Now the existence of the sets $E_{r}$ for any continuum $M$ is an easy consequence of a general theorem of mine [7] on the existence of maximal sets which I shall not take time to discuss here. Suffice it to say that any irreducible carrier $K$ of an essential $\gamma^{r}$ in $M$ is contained in some $E_{r-1}$ and the decomposition into sets $E_{r}$ is always possible. Furthermore, the sets $E_{r}$ are continua and the intersection of any two of them is always a $T_{r}$-set. For example, two $E_{0}$ 's have at most one point in common, two $E_{1}$ 's in the above example have only an arc (which is a $T_{1}$ ) in common. It is no longer true that the $E_{r}$ 's are countable, even for $r=0$. For we are not supposing $M$ locally connected, so that $M$ might consist of a non-enumerable family of concentric circles connected up by a radius; and in this case each of the circles is an $E_{0}$ of $M$. However, corresponding to the property of cyclic elements that the product of each cyclic element $C$ of $S$ by any continuum $N$ in $S$ is itself a continuum, we have an analogous property of the $E_{r}$ 's for any $r$. In the language of homologies this property states that any $\gamma^{0}$ in $C \cdot N$ which is $\sim 0$ in $N$ is $\sim 0$ in $C \cdot N$. Correspondingly (see Whyburn [8]), if $E_{r}$ is any $E_{r}$-set in any continuum $M$ and $N$ is any closed subset of $M$, then any $\gamma^{r}$ in $E_{r} \cdot N$ which is $\sim 0$ in $N$ is $\sim 0$ in $E_{r} \cdot N$. Thus in the above example it will be noted that a 1-cycle in $C$ or $W$ which is $\sim 0$ in $M$ is $\sim 0$ in $C$ or $W$, respectively. Also it is interesting to note that for increasing values of $r$ the decompositions into sets $E_{r}$ are monotone decreasing, that is, each $E_{r}$ is c some $E_{r-1} \subset$ some $E_{r-2} \cdots$ c some $E_{0}$, so that we really do have finer decompositions as $r$ increases. We have seen already that any irreducible carrier of a $\gamma^{r+1}$ is c some $E_{r}$. Thus there exists no closed $(r+1)$-dimensional ring of elements $E_{r}$ just as there existed no closed 1-ring of cyclic elements. Hence, relative to the elements $E_{r}$, the structure of

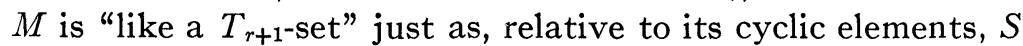
was "dendritic" or "like a $T_{1}$."

6. Applications. We have seen that for the cyclic elements, that is, the $E_{0}$ 's, we have cyclicly extensible and reducible properties. Similarly for the $E_{r}$ 's we have a group of properties 
which are $E_{r}$-extensible and $E_{r}$-reducible, that is, properties $P$ such that

$$
\left(P \text { in each } E_{r}\right) \rightarrow(P \text { in } M),
$$

and conversely.

For example, we saw that if $S \subset R^{2}$ the property of not separating $R^{2}$ is cyclicly extensible and reducible, that is, $E_{0}$-extensible and reducible. Similarly, for any $r \geqq 0$, if $M \subset R^{r+2}$, then the property of not separating $R^{r+2}$ is $E_{r}$-extensible and reducible. Also the property of being locally $\gamma^{s}$-connected for any $s>r$ is $E_{r}$-extensible and reducible. A compact set $N$ is locally $\gamma^{s}$-connected provided that if $\epsilon>0$, a $\delta_{\epsilon}>0$ exists such that any Vietoris cycle $\gamma^{s}$ in $N$ of diameter $\left\langle\delta_{\epsilon}\right.$ is $\sim 0$ in a subset of $N$ of diameter $<\epsilon$. To say that this property is $E_{r}$-extensible and reducible means of course that if each $E_{r}$ is locally $\gamma^{s}$-connected, so also is $M$, and conversely.

Finally, the property of having a vanishing $s$-dimensional Betti number, for any $s>r$, is $E_{r}$-extensible and reducible. This result generalizes the theorem of Borsuk mentioned earlier to the effect that the property of having a zero first Betti number is cyclicly extensible ( $E_{0}$-extensible) in locally connected continua. However, this result in turn is a consequence of a much more inclusive formula by means of which it is possible to express the $s$-dimensional Betti number of any continuum $M$ in terms of the corresponding numbers of the sets $E_{r}$, where $r<s$. In fact, if we denote the $s$-dimensional Betti number of a set $X$ by $p^{s}(X)$, we have simply

$$
p^{s}(M)=\sum p^{s}\left(E_{r}\right), \text { for any } r<s,
$$

the summation being extended over all sets $E_{r}$ in $M$ (see Whyburn [8]). Thus, in particular, we have $p^{s}(M)=\sum p^{s}\left(E_{s-1}\right)$. To see how this formula works, let us take the simple case where $s=1$, and where $M$ is a lemniscate plus a cross bar on one loop. The sets $E_{0}=E_{s-1}$ in $M$ are the loop $L$ and the $\theta$-curve. The formula gives us

$$
p^{1}(M)=p^{1}(L)+p^{1}(\theta)=1+2=3 .
$$

Thus we can obtain the $k$ th Betti numbers of any continuum $M$ simply by adding together the $k$ th Betti numbers of the sets $E_{k-1}$. Now clearly the $E_{r}$-extensibility and reducibility of the 
property of having a zero Betti number of dimension $s$ is obtained merely by setting all the numbers on one side or the other of this equation equal to 0 .

7. A Problem. In connection with the higher order cyclic elements there remains a very fundamental problem which is as yet unsolved, namely, whether or not in a locally $\gamma^{r}$-connected continuum $M$ every element $E_{r}$ carries an essential Vietoris cycle $\gamma^{r+1}$ of dimension $r+1$. In case $r=0$ and in case $M$ is locally $\gamma^{0}$-connected (that is, locally connected in the ordinary sense), the elements $E_{r}$ reduce to the ordinary cyclic elements of $M$ and we have seen that each cyclic element is cyclicly connected. Hence each $E_{0}$ certainly contains a simple closed curve and hence carries an essential $\gamma^{1}$. In the light of known results, this problem may be stated as follows: If a continuum $M$ is locally $\gamma^{r}$-connected and is not separated by any $T_{r}$, does $M$ necessarily carry an essential $\gamma^{r+1}$ ? This is no longer necessarily true even in case $r=0$ if we leave off the condition that $M$ shall be locally connected. For it is well known (see Knaster [1]) that there exists an indecomposable continuum $C$ in the plane having a vanishing first Betti number; and since no indecomposable continuum can have a cut point, $C$ would have no cut point and hence would have only one set $E_{0}$, namely, $C$ itself ; and clearly $C$ carries no essential $\gamma^{1}$.

8. Separating Points and Local Separating Points. Returning to the notion of a cut point, let us consider briefly what happens when we localize this concept. A natural way to do this is simply to say that a point $p$ of a continuum $M$ is a local cut point or a local separating point of $M$, provided $p$ is a cut point of any sufficiently small neighborhood of $p$ in $M$. However, this definition leads to difficulties for it would require that we be able to find arbitrarily small neighborhoods of $p$ such that the part of $M$ in these neighborhoods is connected. This, of course, requires that $M$ be locally connected, and for such sets this definition is perfectly good. For continua in general, however, we need first to extend the notion of a cut point or a separating point of a continuum to an arbitrary set $K$, which we do as follows.

If $K$ is any set, connected or not, a point $p$ of $K$ is called a separating point of $K$, provided that $p$ separates $K$ between 
some two points in the component* $C$ of $K$ containing $p$, that is, we have a separation $K-p=K_{1}+K_{2}$, where $K_{1} \cdot C \neq 0 \neq K_{2} \cdot C$ (compare with Menger [4]). In case $K$ is connected, of course, $C=K$ and the separating points of $K$ are merely the cut points of $K$. It is always true that a separating point $p$ of $K$ is a cut point of the component $C$ of $K$ containing $p$. However, it is not generally true that every cut point of a component $C$ of $K$ is a separating point of $K$. For in the example $K$ consisting of a sequence of intervals converging to a limiting interval, every inner point of the limiting interval is a cut point of that interval but no such point is a separating point of $K$.

Now the concept of a separating point localizes directly and without difficulty. A point $p$ of a continuum $M$ (or of any set $M$ ) is a local separating point of $M$ provided that $p$ is a separating point of some open subset of $M$. From this definition it results easily that any local separating point $p$ of $M$ is a separating point of the part of $M$ in any sufficiently small neighborhood of $p$. For example, any point of a circle or a lemniscate is a local separating point. Any linear graph consists entirely of local separating points plus a finite number of end points.

Since obviously any cut point of a continuum is also a local separating point but not conversely, it follows that the chance of existence of local separating points is much greater than for cut points. It can be proved easily, for example, that in any regular curve, rational curve, or hereditarily locally connected continuum, the local separating points must be everywhere dense (Whyburn [9]), whereas, obviously, there exist curves of these types which have no cut point. There exist very simple curves, for example, a circle, having no cut point, whereas, any locally connected continuum having no local separating point must contain, for any two of its points $a$ and $b$, a set of arcs $[a x b]$ of the power of the continuum each pair of which intersect in just $a+b$ (see Whyburn [11] and Zippin [2]).

The more fundamental theorems on cut points extend with little or no modification to local separating points. Thus the cut point-order theorem extends to give the following theorem.

* By the component of $K$ containing $p$ is meant the maximal connected subset of $K$ containing $p$. 
Local Separating Point-Order Theorem. All save possibly a countable number of the local separating points of any continuum $M$ are points of order 2 of $M$ (Whyburn [11]).

Also, the local separating points of any locally connected set form a Borel set of the class $F_{\sigma}$ and the set of all such points of any continuum is a set of class $G_{\delta \sigma}$ just as was the case with cut points (Whyburn [2]).

So far as has yet been discovered, the local separating points of a continuum $M$ do not yield a useful decomposition of $M$ which is strictly analogous to the cyclic element decomposition of a locally connected continuum. In case they exist in sufficient numbers, however (that is, if they are uncountable), the local separating points of $M$ do yield quite useful decompositions of $M$ of a slightly different sort. To obtain such a decomposition, let $G$ denote the set of all local separating points of $M$ and, for each $p \in M$, let $C(p)$ denote the maximal subcontinuum of $M$ containing $p$ and such that $G \cdot C(p)$ is at most countable. Then the sets $C(p)$ always exist and no two of them which are different can intersect at all. Thus we obtain a decomposition of $M$ into disjoint continua $[C(p)]$. This decomposition is upper semicontinuous in the sense of R. L. Moore $[3,4]$ and its hyperspace $H$, that is, the space whose points are the sets $[C(p)]$, is a regular curve of simple structure. In fact, every subcontinuum of $H$ must contain uncountably many local separating points of $H$ and $H$ is a continuum of finite degree in the sense of Kamiya [1] (see Whyburn [12], [13]).

Still other decompositions by means of local separating points are possible under suitable restrictions on $M$. For example, we may decompose $M$ into maximal subcontinua $D(p)$ of $M$ which have only a countable number of local separating points. However, the possibilities in this direction have not been extensively investigated and herein lies a most interesting and what promises to be a very fruitful unexplored realm of topology, namely, to study intensely the possible decompositions of continua by means of local separating points. It would be most desirable to develop a group of properties which would be extensible from the sets $C(p)$ to the whole continuum $M$ in the same sense that the cyclicly extensible properties extend from the cyclic elements to the whole continuum. 
9. Applications. Among the applications of the local separating point notion to problems in continuum structure, I shall mention three which seem particularly far-reaching.

(i) First we mention an application to the problem of arcwise connectivity of all connected subsets of a continuum. It was shown independently by Moore $[4,5]$ and by Menger $[3]$ that any connected and locally connected $G_{\delta}$-set is arcwise connected. This result lends particular significance to the problem of finding necessary and sufficient conditions in order that every connected subset of a continuum $M$ should be a $G_{\delta}$-set. The solution to this problem is easily given in terms of local separating points. It is embodied simply in the condition that all except a countable number of the points of $M$ shall be local separating points of $M$ (see Whyburn [14]). In fact we may state a more general theorem as follows.

THEOREM. If every connected subset of a continuum $M$ is a Borel set (of any class whatever), then the non-local separating points of $M$ are countable. Conversely, if the non-local separating points of $M$ are countable, then every connected subset of $M$ is locally connected and is both $a G_{\delta}$ and an $F_{\sigma}$.

In conjunction with the Moore-Menger result and the cyclic extensibility of the property of having all connected subsets arcwise connected, this gives the theorem that if the non-local separating points of each true cyclic element of a locally connected continuum $S$ are countable, then every connected subset of $S$ is arcwise connected. Another interesting result of the above theorem is that if each connected subset of $M$ is a Borel set of some class, then every such connected set must be both an $F_{\sigma}$ and $a G_{\delta}$.

(ii) Secondly, the local separating points of a continuum $M$ have a deciding position relative to the existence in $M$ of totally imperfect connected subsets, that is, connected subsets in $M$ which contain no perfect subsets. It has been shown by $F$. Bernstein [1] and Sierpinski [1] that such sets exist in any euclidean space of dimension greater than 1 and by Knaster [2] and Kuratowski that they even exist in the Sierpinski regular curve. This naturally raises the question: Under what conditions will such sets exist in a continuum $M$ ? Again the answer is readily provided in terms of local separating points. For, in order that $M$ contain a totally imperfect connected subset it is neces- 
sary and sufficient that the local separating points of some subcontinuum be countable (Whyburn [15]). In particular it follows from this that the hyperspace $H$ of the decomposition of $M$ above into sets $C(p)$ can contain no totally imperfect connected subset.

(iii) As a final application I will call attention to the natural order basis which the local separating points provide in any regular curve. If $K$ is a regular curve in the Menger-Urysohn sense, a subset $B$ of $K$ is called an order basis for $K$ provided each point $p$ of $K$ is contained in arbitrarily small neighborhoods whose boundaries intersect $K$ in only a finite number of points all of which lie in $B$ and the number of which does not exceed the order of $p$ in $K$. Now we may assert that if $Q$ is any set of local separating points of $K$ which includes all of the at most countable number of local separating points of $K$ of order $>2$ and which is dense in the set of all local separating points of $K$, then $Q$ is an order basis for $K$. Clearly, by virtue of the local separating point-order theorem, such a set $Q$ can always be chosen so that it is countable; and hence every regular curve has a countable order basis consisting entirely of local separating points.

10. A General Problem on Continuous Transformations. I shall devote the time that remains to a consideration of the following general problem concerning the preservation of the structure of a continuum when the continuum undergoes a continuous transformation. Let $A$ and $B$ be continua, and let $T(A)=B$ be a single-valued continuous transformation of $A$ into $B$. The problem is to find conditions on the transformation $T$ and its inverse which will insure that $B$ will be topologically equivalent to $A$, that is, homeomorphic with $A$. In other words, we are asking what sort of continuous transformation will preserve or leave invariant all topological properties of a given continuum $A$. I shall consider only those conditions on $T$ which concern the inverse sets $T^{-1}(b)$ of points on $B$. Thus we are seeking conditions on the sets $T^{-1}(b)$ which will insure that $B$ be topologically equivalent to $A$.

Now the problem has the obvious and trivial solution embodied in the condition that the inverse $T^{-1}$ should also be single-valued, that is, that $T$ be (1-1). For, in the case of compact sets $A$ and $B$, this merely makes $T$ itself a topological transformation. However, in a number of important cases this con- 
dition is known to be stronger than nece ssary. For example, in case $A$ is a simple arc, it is enough, to insure that $B$ also will be an arc, to assume merely that the inverse set $T^{-1}(b)$ for each point $b \epsilon B$ should be connected.

A satisfactory general solution to this problem as I have stated it seems as yet to be considerably beyond our reach. As we shall see later, no such solution may be expected so long as we limit ourselves to conditions on the sets $T^{-1}(b)$ alone; but when we allow conditions both on these sets and on their complements in $A$, the outlook is considerably more hopeful. The principal result up to date in this direction has been obtained by James F. Wardweil. In his dissertation he obtained a condition which is sufficient to make $B$ topologically equivalent to $A$ provided the number of and the condensation of those sets $\left[T^{-1}(b)\right]$ which are non-degenerate also are suitably restricted.

Aside from Wardwell's work, practically all other progress which has been made on this problem is confined to particular types of sets $A$; and concerning these types a rich collection of theorems has been proved. I have already mentioned the case where $A$ is a simple arc, and the condition in this case is simply that the inverse sets $\left[T^{-1}(b)\right]$ should all be continua. Now a continuous transformation satisfying this condition, that is, such that $T^{-1}(b)$ is connected for every $b \epsilon B$, has been called (see Morrey [1]) a monotone transformation. The term seems appropriate because of the analogy with the case of a real function $y=f(x)$; for if $f(x)$ is monotone in the usual sense, then for each value $y_{1}$ of $y$ the set of values $x_{1}$ of $x$ such that $f\left(x_{1}\right)=y_{1}$ is always connected. It is interesting to note that a continuous transformation $T(A)=B$, where $A$ and $B$ are compact, will be monotone if and only if the property of connectedness is invariant under $T^{-1}$.

In case $A$ is an arc, then the solution to our problem is embodied in the condition that the transformation $T$ be monotone. The same is true in case $A$ is a circle or any simple closed curve. Thus we have the following result.

THEOREM. If $A$ is a simple arc $\{$ simple closed curve $\}$ and $T(A)=B$ is monotone, then $B$ is a simple arc simple closed curve $\}$ or a single point.

11. Monotone Transformations on the Sphere. Cactoids. Now 
when we take $A$ to be a sphere (that is, the surface of a sphere), it appears at once that the condition that $T$ be a monotone is no longer sufficient to make $B$ homeomorphic with $A$. For we can transform a sphere $A$ into a set $B$ consisting of two tangent spheres by sending the equator on $A$ into the point of tangency and mapping each of the two hemispheres onto the two spheres minus one point. Similarly, by sending two circles on $A$ into points, we can map it onto three tangent spheres, or we can map it into a diameter by a simple projection which clearly is a monotone transformation. Thus, if we are to have $B$ topologically equivalent to $A$, extra conditions must be added. Now it will be noted that in each of the cases here illustrated where $B$ is not a topological sphere, some of the sets $T^{-1}(b)$ separate $A$. In the first case, the inverse of the point of tangency of the two spheres is the equator of the given sphere and hence cuts it into two parts. In the last case, all sets $T^{-1}(b)$ except two cut $A$. Thus we are led to the following theorem due to R. L. Moore [3]; it solves our problem in the case of the sphere.

Theorem. If $A$ is a sphere, if $T$ is monotone and no set $T^{-1}(b)$ separates $A$, and if $B$ contains more than one point, then $B$ is homeomorphic with $A$.

Moore states this theorem in terms of upper semi-continuous decompositions of a sphere (or plane) into continua rather than in terms of a continuous transformation as I have done. It was shown later by Alexandroff [1] and by Kuratowski [3] that any upper semi-continuous decomposition of a compact space is equivalent to a continuous transformation defined on that space; and hence we can use the language of continuous transformations to describe this and other results some of which were originally stated in terms of other notions.

The case of the sphere (or plane) is of such interest and usefulness in connection with the study of surfaces both from a topological standpoint and an analytical standpoint that it may be worthwhile to consider briefly some results which have been found in connection with this theorem. In the first place we have already seen that assuming $T$ monotone was not sufficient, in the case of the sphere, to make $B$ topologically equivalent to $A$. However, suppose we investigate the possible images of $A$ when we do just assume $T$ monotone. We have seen that we may 
get images such as a string of tangent spheres, an arc, two spheres joined by an arc. Now from the point of view of cyclic elements, these various possibilities are very similar. In fact in each case every true cyclic element of $B$ is a topological sphere. This is not accidental; indeed it is a characteristic property for images of a sphere under monotone transformations. Thus we have the following theorem which is due to R. L. Moore [2].

Theorem. If $A$ is a sphere and $T$ monotone, then every true cyclic element of $B$ is a topological sphere.

The very picturesque name cactoid has been used to describe such a set $B$, that is, a cactoid is a locally connected continuum every true cyclic element of which is a topological sphere. Now the cactoids form a well defined mathematical class which is equivalent to the class of all monotone transformations definable on the sphere. That is, not only is the image of any sphere under any monotone transformation always a cactoid, but also any cactoid is always the image under some monotone transformation of the sphere (see Moore [2]). Also this class is closed under the operation of taking images under monotone transformations, by the following theorem. (Whyburn [16].)

THEOREM. The image of any cactoid under any monotone transformation is itself a cactoid.

12. Non-alternating Transformations on the Circle. Boundary Curves. The relation of the cactoids to the sphere suggests that there should be a class of one-dimensional curves which is analogously related to the circle. This is indeed true as we shall soon see. Since a cactoid is a locally connected continuum every true cyclic element of which is a topological sphere, its onedimensional analog, therefore, is a locally connected continuum every true cyclic element of which is a topological circle. We have called such a curve a boundary curve due to the fact (see Ayres [4]) that it is also characterized by the property that it is always homeomorphic with the boundary of a plane domain. We now ask: What kind of a transformation will produce a boundary curve from a circle? Of course, a monotone transformation will do so, since it always produces a topological circle from a circle; but given a boundary curve, in general it cannot of course be obtained from a circle by a monotone transformation. 
The answer is given by the condition on the sets $T^{-1}(b)$ that they not separate each other on the circle $A$. This means that for no two points $b_{1}$ and $b_{2}$ of $B$ will $T^{-1}\left(b_{1}\right)$ separate any two points of $T^{-1}\left(b_{2}\right)$ on $A$. A transformation having this property is called a non-alternating transformation (Whyburn [16]), since in the case of the circle $A$ it simply means that as we move around the circle $A$ we never can meet alternately points of two distinct sets $T^{-1}(b)$. Thus we have the following theorem.

TheOREm. The image of every circle under any non-alternating transformation is a boundary curve.

The converse of this is also true, namely, any boundary curve $B$ is the image under some non-alternating transformation of a circle. Thus, given in particular any locally connected continuum $B$ bounding a plane domain, we can map the circle $A$ onto $B$ by a non-alternating transformation. It is interesting to note that this can always at the same time be done in a certain minimal way from the standpoint of multiplicity. That is, we can map the circle $A$ onto any boundary curve $B$ by a nonalternating transformation $T$ in such a way that for each $b \in B$, the number of points in $T^{-1}(b)$ is exactly the same as the number of components into which $p$ cuts $B$, provided either of these numbers is finite. Finally, just as the class of cactoids is closed under monotone transformations, so also is the class of boundary curves closed under non-alternating transformations, which means simply that the image of any boundary curve under any non-alternating transformation is itself a boundary curve.

13. The 3-Space. Returning to our problem of finding conditions on the sets $T^{-1}(b)$ which will make $B$ homeomorphic with $A$, we have seen that the solution has been found for the case of the circle and of the sphere. It is clear that the results above stated could easily be modified so as to yield the solution when $A$ is the ordinary line or plane. Let us next consider the case of the 3-dimensional space. We may expect of course that extra conditions on the sets $T^{-1}(b)$ may be necessary. Just how much more may be necessary, however, no one is able at present to say, since the problem for this case is still unsolved. The difficulties met here may be illustrated by some simple considerations. Suppose we let $A$ be the 3-space and let us take 
the simple case where one single arc $x y$ in $A$ goes into a point $b$ of $B$ but where every other point of $B$ comes from a single point of $A$, that is, $T(x y)=b$ but $T$ is (1-1) on $A-x y$. Even in this case we cannot say that $B$ is homeomorphic with $A$. If $x y$ is a linear interval this will indeed be true. But Antoine [1] has shown that there are arcs $x y$ in 3-space $A$ which are knotted in the sense that $A-x y$ is not homeomorphic with $A$ minus a linear interval. Thus in the example just given, if $x y$ is such an arc, then since $B-b$ is homeomorphic with $A-x y$, we see that $B$ cannot be a topological 3-space; because if it were, $B-b$ would be homeomorphic with $B$ minus a linear interval.

14. A Trial Condition. The example just given shows clearly that if we limit ourselves to topological conditions on the sets $T^{-1}(b)$ alone, no satisfactory solution to our problem is possible for the case where $A$ is a euclidean space of dimension three or greater. The condition imposed above in the case of the sphere, that the sets not separate $A$, may of course be stated as an intrinsic property of the sets by using the notion of higher connectivity.

However, when the conditions on both the sets $T^{-1}(b)$ and their complements are allowed, the outlook is more hopeful. Suppose for instance we impose the condition that for each $b \epsilon B$ the complement of every set $T^{-1}(b)$ in $A$ be homeomorphic with the complement of every single point in $A$. This condition will rule out at once the possibility encountered in the example and described in $\S 13$, that is, if any set $T^{-1}(b)$ is an arc in $A$, where $A$ is the 3 -space, then this arc cannot be knotted in the sense described above. However, in the cases of the circle and the sphere it is to be noted that it reduces to exactly the conditions appropriate to these respective cases. For, if $A$ is a circle, then to say that the complement of a closed subset $K$ of $A$ is homeomorphic with the complement of a point is exactly the same as saying that $K$ is connected. Also, if $A$ is a sphere, then the condition that a closed set on $A$ be connected and not separate $A$ is equivalent to the condition that its complement be homeomorphic with the complement of a point. Whether this condition actually will be sufficient to make $B$ homeomorphic with $A$ in general or in the case of the 3 -space, is not yet known. It is being investigated at present and some results have been ob- 
tained using it; so that one can at least hope that it or some of its many possible modifications may lead to a solution to the problem, if not in general, at least in a number of the more interesting particular cases such as the ones I have indicated.

\section{BIBLIOGRAPHY}

AlexandrofF, P.

1. Über stetige Abbildungen kompakter Räume, Mathematische Annalen, Antorne, L. vol. 96 (1926), pp. 555-571.

1. Sur les voisinages de deux figures homéomorphes, Fundamenta Mathematicae, vol. 5 (1924), pp. 265-287.

Ayres, W. L.

1. Concerning continuous curves in metric space, American Journal of Mathematics, vol. 51 (1929), pp. 577-594.

2. Concerning the arc-curves and basic sets of a continuous curve, Transactions of this Society, vol. 30 (1928), pp. 567-578, and vol. 31 (1929), pp. 595-612.

3. Some generalizations of the Sherrer fixed-point theorem, Fundamenta Mathematicae, vol. 16 (1930), pp. 332-336.

4. Continuous curves homeomorphic with the boundary of a plane domain, Fundamenta Mathematicae, vol. 14 (1929), pp. 92-95.

Bernstein, F.

1. Leipziger Berichte, vol. 60 (1908), p. 329.

Borsuk, K.

1. Über eine Klasse von lokal zusammenhängenden Räumen, Fundamenta Mathematicae, vol. 19 (1932), p. 230.

2. Einige Sätze über stetige Streckenbilder, Fundamenta Mathematicae, vol. 18 (1932), pp. 198-213.

Gehman, H. M.

1. Concerning end points of continuous curves and other continua, Transactions of this Society, vol. 30 (1928), pp. 63-84.

Kamiya, $\mathrm{H}$.

1. Tôhoku Mathematical Journal, vol. 36 (1933), pp. 58-72.

KLINE, J. R.

1. Separation theorems and their relation to recent developments in analysis situs, this Bulletin, vol. 34 (1928), pp. 155-192.

KNASTER, B.

1. Un continu dont tout sous-continu est indécomposable, Fundamenta Mathematicae, vol. 3 (1922), pp. 247-286.

2. (With C. Kuratowski) $A$ connected and connected im kleinen point set which contains no perfect subset, this Bulletin, vol. 33 (1927), pp. 106109.

Kuratowski, C.

1. (With G. T. Whyburn) Sur les éléments cycliques et leurs applications, Fundamenta Mathematicae, vol. 16 (1930), pp. 305-331. 
2. Quelques applications d'élêments cycliques de $M$. Whyburn, Fundamenta Mathematicae, vol. 14 (1929), pp. 138-144.

3. Sur les dêcompositions semi-continues d'espaces métriques compacts, Fundamenta Mathematicae, vol. 11 (1928), pp. 169-185.

MAZURKIEWICZ, S.

1. Un thêorème sur les lignes de Jordan, Fundamenta Mathematicae, vol. 2 (1921), pp. 119-130.

Menger, $\mathrm{K}$.

1. Grundzüge einer Theorie der Kurven, Mathematische Annalen, vol. 95 (1925), pp. 272-306.

2. Kurventheorie, B. G. Teubner, 1932.

3. Zur Begründung einer axiomatischen Theorie der Dimension, Monatshefte für Mathematik und Physik, vol. 36 (1926), pp. 193-218.

4. Remarks concerning the paper of W. L. Ayres on the regular points of a continuum, Transactions of this Society, vol. 33 (1931), pp. 663-667.

MOORE, R. L.

1. Concerning cut points of continuous curves and of other closed and connected point sets, Proceedings of the National Academy of Sciences, vol. 9 (1923), pp. 101-106.

2. Concerning upper semi-continuous collections, Monatshefte für Mathematik und Physik, vol. 36 (1929), pp. 81-88.

3. Concerning upper semi-continuous collections of continua, Transactions of this Society, vol. 27 (1925), pp. 416-428.

4. Foundations of Point Set Theory, American Mathematical Society Colloquium Publications, 1932.

5. Abstract sets and the foundations of analysis situs (abstract), this Bulletin, vol. 33 (1927), p. 141.

Morrey, C. B.

1. The topology of (path) surfaces, American Journal of Mathematics, vol. 57 (1935), pp. 17-50.

SHERRER, W.

1. Über ungeschlossene stetige Kurven, Mathematische Zeitschrift, vol. 24 (1925), pp. 125-130.

SiERPINSKI, W.

1. Sur un ensemble punctiforme connexe, Fundamenta Mathematicae, vol. 1 (1920), pp. 7-10.

URYSOHN, P.

1. Mémoire sur les multiplicités cantoriennes. Deuxième partie Verhandelingen der koninklijke Akademie van Wetenshappen te Amsterdam, vol. 13 (1928), No. 4.

VIETORIS, L.

1. Über den höheren Zusammenhang kompakter Räume und eine Klasse von zusammenhangstreuen Abbildungen, Mathematische Annalen, vol. 97 (1927), pp. 454-572.

WhyBuRN, G. T.

1. Concerning cut points of continua, Transactions of this Society, vol, 30 (1928), pp. 597-609. 
2. Cut points of connected sets and of continua, ibid., vol. 32 (1930), pp. 147-154.

3. Concerning the structure of a continuous curve, American Journal of Mathematics, vol. 50 (1928), pp. 167-194.

4. Cyclicly connected continuous curves, Proceedings of the National Academy of Sciences, vol. 13 (1927), pp. 31-38.

5. Concerning Menger regular curves, Fundamenta Mathematicae, vol. 12 (1928), pp. 264-294.

6. The rationality of certain continuous curves, this Bulletin, vol. 36 (1930), pp. 522-524.

7. Concerning maximal sets, this Bulletin, vol. 40 (1934), pp. 159-164.

8. Cyclic elements of higher orders, American Journal of Mathematics, vol. 56 (1934), pp. 133-146.

9. On regular points of continua and regular curves of at most order $n$, this Bulletin, vol. 35 (1929), pp. 218-224.

10. Continuous curves without local separating points, American Journal of Mathematics, vol. 53 (1931), pp. 163-166.

11. Local separating points of continua, Monatshefte für Mathematik und Physik, vol. 36 (1929), pp. 305-314.

12. Decompositions of continua by means of local separating points, American Journal of Mathematics, vol. 55 (1933), pp. 437-457.

13. Concerning continua of finite degree and local separating points, ibid, vol. 57 (1935), pp. 11-16.

14. Sets of local separating points of a continuum, this Bulletin, vol. 39 (1933), pp 97-100.

15. On the existence of totally imperfect and punctiform connected subsets in a given continuum, American Journal of Mathematics, vol. 55 (1933), pp. 146-152.

16. Non-alternating transformations, ibid, vol. 56 (1934), pp. 294-302. WILDER, R. L.

1. Concerning continuous curves, Fundamenta Mathematicae, vol. 7 (1925), pp. 340-377.

2. Point sets in three and higher dimensions and their investigation by means of unified analysis situs, this Bulletin, vol. 38 (1932), pp. 649-692.

ZARANKIEWICZ, C.

1. Sur les points de division dans les ensembles connexes, Fundamenta Mathematicae, vol. 9 (1927), pp. 124-171. ZIPPIN, L.

1. On continuous curves and the Jordan curve theorem, American Journal of Mathematics, vol. 52 (1930), pp. 331-350.

2. Note on locally cyclicly connected continua (abstract), this Bulletin, vol. 36 (1930), p. 805.

The University of Virginia 